\title{
Osteomyelitis presenting as genital sore: a case report
}

\author{
V L Rege, P Shukla
}

\begin{abstract}
A female attending the skin outpatient department presented with a genital sore which was treated as chancroid. On further investigation it proved to be the opening of a sinus secondary to chronic osteomyelitis of the pubic symphysis
\end{abstract}

(Genitourin Med 1993;69:460-461)

\section{Introduction}

Genital sores are a common presentation of sexually transmitted diseases (STDs) in tropical countries. At times they may be due to nonveneral conditions. We report one such case initially thought to be an STD but later turned out to be a genital ulcer secondary to chronic osteomyelitis of the pubic symphysis. Such a case has not been described in the literature to the best of our knowledge.

\section{Case report}

A 32 year old female attended the skin outpatient department at Goa Medical College with a genital sore of one week's duration. She had been married for the past 12 years and had five children. All deliveries were at term and normal except the last delivery, 2 years previously which was rather difficult. Immediately following delivery she noticed a dull pain and tenderness in her lower abdomen which worsened on walking and had continued for the past 2 years. Both husband and wife denied having pre or extramarital sexual exposure.

One month previously she had noticed a slowly growing moderately painful papulopustular lesion on the lateral border of the left labia majora. It broke down 3 weeks later forming a dirty necrotic tender ulcer $3.5 \mathrm{~cm}$ in diameter with irregular ragged borders. It was fixed to the underlying tissues and exuded a purulent discharge which increased on manipulation. The left sided inguinal glands in the horizontal chain were enlarged, soft, matted, tender with normal overlying skin. The smear from discharge showed Gram positive cocci in groups and chains but was negative for treponemes, $H$. Ducreyi, AFB, Donovan bodies and fungal elements. Based on the clinical findings the patient was diagnosed as chancroid and treated with cotrimoxazole, 4 tablets per day. At a follow up visit 10 days later the discharge showed a slight decrease and a sinus was revealed.

Through this sinus a sinogram was per- formed. It showed a draining sinus originating at the joint cavity of pubic symphysis, to open at the site of the ulcer. In addition there was dislocation of the joint with destruction of joint surfaces which extended to involve the superior and inferior pubic rami (fig).

Culture studies from the discharge revealed growth of Staphylococcus Aureus and group B streptococci sensitive to cephelexin, chloramphenicol and amoxycillin. No growth of AFB or fungi was noted. The other investigations were as follows: haemoglobin $10 \mathrm{~g} /$ $100 \mathrm{ml}$; total WBC count, $11500 / \mathrm{mm}^{3}$; differential WBC count-neutrophils $52 \%$, lymphocytes $46 \%$ and eosinophils $2 \%$, ESR-45 $\mathrm{mm}$ in first hour. The VDRL was non reactive, ELISA for HIV antibodies negative and an IVP normal.

The final diagnosis was chronic osteomyelitis of the symphysis pubis with a draining sinus and ulcer. The patient was given oral cephelexin $2 \mathrm{~g}$ per day for one month. This resulted in complete healing, although an atrophic hypopigmented scar was left at the site of the ulcer.

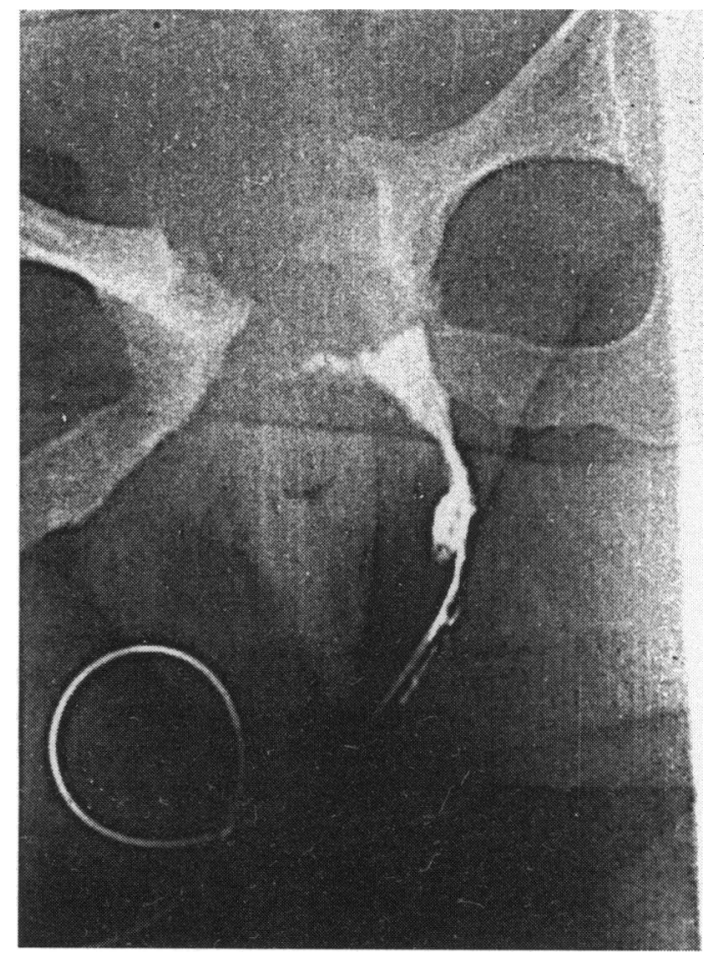

Figure Radiograph showing a sinus starting at joint space of symphysis pubis and opening at the site of the genital ulcer. In addition there is destruction and dislocation of pubic symphysis. 


\section{Discussion}

This case presented as a genital ulcer which on closer examination was found to be a discharging sinus. This prompted us to do a sinogram revealing the underlying pathology. With appropriate antibiotics and bed rest the pain in the lower abdomen and underlying osteomyelitis was controlled with full healing of the ulcer and sinus. The patient did not want further treatment and was lost to follow up.

It is known that there is relaxation and weakening of pelvic joints during pregnancy. During difficult deliveries especially with instrumentation or prolonged labour there is a chance of injury to pubic symphysis. ${ }^{12}$
Coupled with this, incomplete tearing of the ligaments connecting the symphysis, associated haemorrhage can take place with excessive abduction of the thighs. Arthritis and osteomyelitis are possible complications.

In our patient the pain, experienced after the fifth delivery and which continued could be explained on the basis of the above pathology which subsequently led to osteomyelitis and a discharging sinus.

1 Harry O. Oxorn-foote. Human Labor and Birth; 5th ed East Norwalk, USA. Prentis Hall Inc; 1986;552-3.

2 Myerscough PR. Munro Kerrs Operative Obstetrics; 10th ed; London. Bailliere Tindall; 1982;465-6. 